\title{
Thermally Stimulated Depolarization Currents in $\mathrm{NaCl}: \mathrm{Ca}^{2+}$ Crystals
}

\author{
J. POŹNIAK \\ W. Trzebiatowski Institute of Low Temperature and Structure Research \\ Polish Academy of Sciences \\ P.O. Box 937, 50-950 Wrocław, Poland \\ (Received June 7, 2004; revised version September 27, 2004; \\ in final form January 28, 2005) \\ Analysis of the thermally stimulated depolarization currents in \\ $\mathrm{Ca}^{2+}$-doped $\mathrm{NaCl}$ crystals is carried out by taking into account the pres- \\ ence of the $\mathrm{Ca}^{2+}$-cation vacancy complexes with vacancies in both nearest- \\ -neighbourhood and next-nearest neighbourhood position. Relaxation pa- \\ rameters are derived by fitting the experimental data to standard equations. \\ The results are, where possible, compared with the relevant parameters re- \\ ported in the literature.
}

PACS numbers: 77.22.Ej

\section{Introduction}

Relaxation phenomena related to the electric field induced orientation of $\mathrm{Me}^{2+}$ impurity cation-cation vacancy (I-V) complexes in alkali halide crystals have been intensively investigated since long time. The presence and properties of these complexes were examined among others by measurements of the thermally stimulated depolarization (TSD) currents (see e.g. [1, 2]).

Theoretical foundations of the TSD method were elaborated by Bucci and Fieschi [3] who anticipated the existence of the I-V complexes in which the cation vacancy is in the nearest neighbourhood (nn state) of the $\mathrm{Me}^{2+}$ cation. In this case the TSD current is described by an appropriate equation with one relaxation time and two activation energies. In some instances this theory proved to be successful (see e.g. [1, 2, 4-9]). The reason of this accordance could be the existence of dominant elementary jumps, which contribute to the depolarization. 
However, in some instances this theory did not work [10-18] suggesting the existence of a noticeable contribution of the $\mathrm{I}-\mathrm{V}$ complexes in which the cation vacancy is in the next-nearest neighbourhood (nnn state) of the $\mathrm{Me}^{2+}$ cation. In these cases the experimental TSD peak in fact consists of two overlapping peaks and is characterized by two relaxation times [15-18].

The required equation describing the TSD current related to the reorientation of $\mathrm{nn}$ and nnn type of $\mathrm{I}-\mathrm{V}$ complexes in alkali halide crystals is known [18]. This equation involves a set of possible elementary jumps which contribute to the depolarization. Unfortunately, only for $\mathrm{NaCl}: \mathrm{Mn}^{2+}$ crystals the appropriate elementary jump frequencies are given in the literature [19].

The purpose of the present paper is to report the newest results concerning the behaviour of the dipolar TSD-peaks of $\mathrm{NaCl}: \mathrm{Ca}^{2+}$ crystals, and to discuss the properties of these peaks in terms of above mentioned models.

\section{Theory}

If it is assumed that in each $\mathrm{I}-\mathrm{V}$ complex the vacancy occupies an nn site relative to a divalent ion, the equation for TSD current density is $[2,3]$ :

$$
j(T)=P_{0} \tau^{-1}(T) \exp \left[-\frac{1}{b} \int_{T_{\mathrm{p}}}^{T} \tau^{-1}\left(T^{\prime}\right) \mathrm{d} T^{\prime}\right],
$$

where

$$
P_{0}=\frac{2}{3} \frac{C_{i} \mu^{2}}{k T_{\mathrm{p}}} E_{\mathrm{p}}
$$

$C_{i}$ is the number of $\mathrm{I}-\mathrm{V}$ complexes per unit volume, $\mu$ - their electric moment, $E_{\mathrm{p}}$ - the polarizing electric field, $T_{\mathrm{p}}$ - the effective polarizing temperature, $T$ the absolute temperature of the crystal, $b$ - the linear heating rate and $\tau(T)-$ the relaxation time. $\tau(T)$ is related to the elementary jump frequencies $\omega_{1}$ and $\omega_{2}$ that respectively characterize the movement of the vacancy about impurity and direct interchange of the vacancy and the impurity. Their interdependence is

$$
\tau(T)=1 /\left[2\left(\omega_{1}+\omega_{2}\right)\right] .
$$

Usually $\omega_{1} \gg \omega_{2}$. For this reason the analyses of TSD data are based on the Arrhenius type dependence of the relaxation time

$$
\tau(T)=1 / 2 \omega_{1}=\tau_{0} \exp (E / k T),
$$

where $\tau_{0}=$ const, $E$ is the activation energy and $k$ - the Boltzmann constant. In this case the equation for the TSD current density is

$$
j(T)=P_{0} \tau_{0}^{-1} \exp \left(-\frac{E}{k T}\right) \exp \left[-\frac{1}{b \tau_{0}} \int_{T_{\mathrm{p}}}^{T} \exp \left(-\frac{E}{k T^{\prime}}\right) \mathrm{d} T^{\prime}\right] .
$$

The first exponential is responsible for the initial increase in the current with 
temperature, while the second exponential, which dominates at high temperatures, gradually slows down the current rise and then depresses it rapidly. The first exponential dominates in the low-temperature range so that

$$
j(T) \simeq P_{0} \tau_{0}^{-1} \exp \left(-\frac{E}{k T}\right) .
$$

In this case the plot of $\ln j(T)$ as a function of $1 / T$ is a straight line from the slope of which the value of $E$ can be calculated (initial rise method). Next, the curve that plots TSD current versus temperature has a maximum at

$$
T_{\mathrm{m}}=\left[\frac{E}{k} b \tau_{0} \exp \left(\frac{E}{k T_{\mathrm{m}}}\right)\right]^{\frac{1}{2}},
$$

so that the value of $\tau_{0}$ can be calculated.

Moreover, the theoretical studies of the TSD currents in $\mathrm{Me}^{2+}$ doped $\mathrm{NaCl}$-type alkali halide crystals were performed by taking into account the presence of the $\mathrm{I}-\mathrm{V}$ complexes with vacancies in both nn and nnn positions [16-18]. In this case the equation for TSD current density is

$$
j(T)=\frac{A_{1}}{\tau_{1}(T)} \exp \left[\frac{1}{b} \int_{T_{\mathrm{p}}}^{T} \frac{\mathrm{d} T^{\prime}}{\tau_{1}\left(T^{\prime}\right)}\right]+\frac{A_{2}}{\tau_{2}(T)} \exp \left[\frac{1}{b} \int_{T_{\mathrm{p}}}^{T} \frac{\mathrm{d} T^{\prime}}{\tau_{2}\left(T^{\prime}\right)}\right],
$$

where

$$
\begin{aligned}
& \tau_{1}^{-1}(T)=\lambda_{1}=-\left(\omega_{1}+\omega_{2}+2 \omega_{3}+\omega_{4}\right)+\sqrt{\Delta} / 2, \\
& \tau_{2}^{-1}(T)=\lambda_{2}=-\left(\omega_{1}+\omega_{2}+2 \omega_{3}+\omega_{4}\right)-\sqrt{\Delta} / 2, \\
& \sqrt{\Delta}=2\left[\left(\omega_{1}+\omega_{2}-2 \omega_{3}+\omega_{4}\right)^{2}+4 \omega_{3} \omega_{4}\right]^{1 / 2},
\end{aligned}
$$

$\omega_{3}$ is the jump frequency of nnn vacancy to an adjacent nn site and $\omega_{4}$ - the jump frequency for the opposite process. $A_{1}$ and $A_{2}$ are the constants, which can be presented in the form

$$
\begin{aligned}
& A_{1}=-\left.\mu \frac{a_{1}}{\omega_{3}}\left[2\left(\omega_{1}+\omega_{2}+\omega_{4}\right)+\left(\lambda_{1}-\lambda_{2}\right)\right]\right|_{T=T_{\mathrm{p}}}, \\
& A_{2}=-\left.\mu \frac{a_{2}}{\omega_{3}}\left[2\left(\omega_{1}+\omega_{2}+\omega_{4}\right)-\left(\lambda_{1}-\lambda_{2}\right)\right]\right|_{T=T_{\mathrm{p}}},
\end{aligned}
$$

where

$$
\begin{aligned}
& a_{1}=\left.\frac{1}{3} C_{i} x \frac{\omega_{3}}{2 \omega_{3}+\omega_{4}}\left[1+2 \frac{\left(2 \omega_{3}+\omega_{4}\right)-\left(\omega_{1}+\omega_{2}\right)}{\lambda_{1}-\lambda_{2}}\right]\right|_{T=T_{\mathrm{p}}}, \\
& a_{2}=\left.\frac{1}{3} C_{i} x \frac{\omega_{3}}{2 \omega_{3}+\omega_{4}}\left[1-2 \frac{\left(2 \omega_{3}+\omega_{4}\right)-\left(\omega_{1}+\omega_{2}\right)}{\lambda_{1}-\lambda_{2}}\right]\right|_{T=T_{\mathrm{p}}},
\end{aligned}
$$


$x=e a E_{\mathrm{p}} / 2 k T_{\mathrm{p}}, e$ is the elementary electron charge and $a$ - the distance between neighbouring anion and cation (half the lattice parameter).

The elementary jump frequencies are assumed to follow a simple Arrhenius dependence

$$
\omega_{i}=\omega_{0 i} \exp \left(-E_{i} / k T\right) \quad(i=1,2,3,4) .
$$

\section{Experimental procedure}

Single $\mathrm{NaCl}: \mathrm{Ca}^{2+}$ crystal grown in vacuum sealed quartz ampoule by the Bridgman method from pre-purified material, treated in the melt with $\mathrm{CCl}_{4}$ saturated argon, was used.

The samples (area: 0.6 to $1 \mathrm{~cm}^{2}$, thickness: 0.8 to $2 \mathrm{~mm}$ ) were cleaved along the (100) faces. Prior to measurements each sample was quenched to room temperature after $30 \mathrm{~min}$ annealing at $500^{\circ} \mathrm{C}$. Next, the samples were mounted in a holder and placed in a cryostat which was evacuated to $10^{-5}$ Torr. The crystal holder was supplied with an electric heater and electronic controller permitting to keep the temperature constant at the desired value $T$ with an accuracy of $\pm 0.5 \mathrm{~K}$. The samples were polarized in a constant electric field $E_{\mathrm{p}}=1.7 \times 10^{4} \mathrm{~V} / \mathrm{cm}$ for $t_{\mathrm{p}}=3 \mathrm{~min}$ at temperature $T_{\mathrm{p}}=218 \mathrm{~K}$ after which the field was switched off.

The samples were then warmed up at nearly constant rate: $b_{1}=$ $2.16(6) \times 10^{-2} \mathrm{~K} / \mathrm{s}, b_{2}=4.3 \times 10^{-2} \mathrm{~K} / \mathrm{s}$, or $b_{3}=8.5 \times 10^{-2} \mathrm{~K} / \mathrm{s}$. The TSD currents were measured with WK-16 electrometer (the noise level of our electric circuit was $5 \times 10^{-16} \mathrm{~A}$ ). The temperature was determined by means of a copperconstantan thermocouple.

The concentration of the $\mathrm{Ca}^{2+}$ ions in samples examined (determined by means of atomic absorption spectroscopy) was equal to about $120 \mathrm{ppm}$.

\section{Results and discussion}

Figure 1 shows, among other, the experimentally determined TSD bands (points: $\circ, \Delta, \bullet$ ) obtained for quenched samples. All values of $j(T)$ are normalized to the maximum intensities $j\left(T_{\mathrm{m}}\right)$. The analysis which was performed on these bands by the initial rise method is presented in Fig. 2. The low-temperature parts of $\ln j(T)$ vs. $1 / k T$ plots are well represented by straight lines from the slope of which the value $E=0.69 \mathrm{eV}$ has been calculated. This value lies between the experimental ones reported by different authors: $0.67 \mathrm{eV}$ [3], $0.678 \mathrm{eV}[6]$, $0.68 \mathrm{eV}$ [20], $0.695 \mathrm{eV}$ [21], $0.70 \mathrm{eV}$ [22] and $0.702 \mathrm{eV}$ [23]. Using value $E=0.69 \mathrm{eV}$ and the experimental values of $T_{\mathrm{m}}$ and $b$ together with Eq. (5) we find $\tau_{0}^{-1}=$ $1.1408 \times 10^{14} \mathrm{~s}^{-1}$. This value is in close agreement with value $1.2 \times 10^{14} \mathrm{~s}^{-1}$ reported by Dansas [21]. The concentration of $\mathrm{I}-\mathrm{V}$ complexes in samples examined was evaluated by the relation 


$$
P_{0}=\int_{0}^{\infty} j\left(T^{\prime}\right) \mathrm{d} T^{\prime}
$$

where the integral is actually the area of the TSD peak and $P_{0}$ is given by Eq. (1a). The calculations were performed for effective dipole moment $\mu$ equal to 2.83 eA [24]. The result of the numerical calculations is $C_{i}=(33 \pm 1) \mathrm{ppm}$.

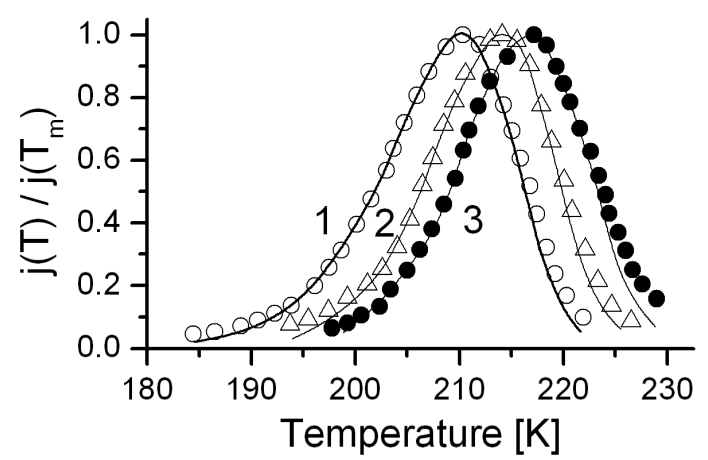

Fig. 1. Comparison between experimental (points: $\circ, \Delta, \bullet$ ) and calculated (full lines) TSD-current bands for the $\mathrm{NaCl}: \mathrm{Ca}^{2+}$ crystals. Experimental data: $T_{\mathrm{p}}=218 \mathrm{~K}$, $t_{\mathrm{p}}=3 \mathrm{~min}, E_{\mathrm{p}}=1.8 \times 10^{4} \mathrm{~V} / \mathrm{cm}$. Curve $1: b_{1}=2.16(6) \times 10^{-2} \mathrm{~K} / \mathrm{s}, T_{\mathrm{m}}=210.5 \mathrm{~K}$, $j\left(T_{\mathrm{m}}\right)=9.96 \times 10^{-14} \mathrm{~A} / \mathrm{cm}^{2}$. Curve $2: b_{2}=4.3 \times 10^{-2} \mathrm{~K} / \mathrm{s}, T_{\mathrm{m}}=215 \mathrm{~K}$, $j\left(T_{\mathrm{m}}\right)=1.84 \times 10^{-13} \mathrm{~A} / \mathrm{cm}^{2} . \quad$ Curve $3: b_{3}=8.5 \times 10^{-2} \mathrm{~K} / \mathrm{s}, T_{\mathrm{m}}=218.5 \mathrm{~K}$, $j\left(T_{\mathrm{m}}\right)=3.57 \times 10^{-13} \mathrm{~A} / \mathrm{cm}^{2}$.

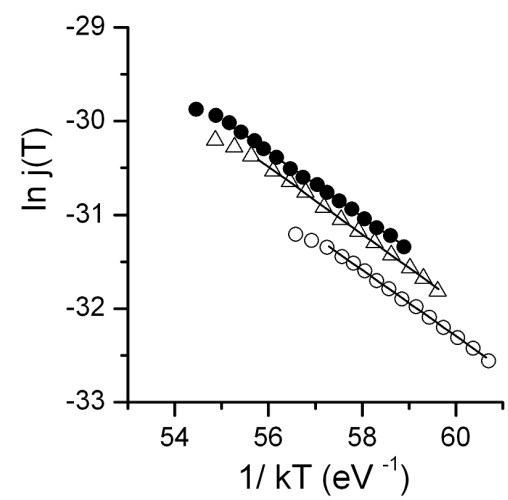

Fig. 2. Analysis of the experimental TSD-current bands shown in Fig. 1 by the initial rise method.

Next, we have assumed that the relaxation frequency can be approximated by the Arrhenius-like equation

$$
-\lambda_{1}=\omega_{1}+\omega_{2}+2 \omega_{3}+\omega_{4}-\sqrt{\Delta} / 2 \simeq \tau_{0}^{-1} \exp (-E / k T),
$$

where $\omega_{i}$ are given by Eqs. (10). This assumption is justified later. Moreover, we 
have assumed that

$$
\omega_{2}=2.3 \times 10^{13} \exp (-0.96 \mathrm{eV} / k T) \quad\left[\mathrm{s}^{-1}\right] .
$$

This information has been obtained from measurements of the diffusion coefficient of the impurity ions $\mathrm{Ca}^{2+}[21,25]$.

The method of least-squares fitting of Eq. (11) to the reorientation energy $E=0.69 \mathrm{eV}$ and to $\tau_{0}^{-1}=1.1408 \times 10^{14} \mathrm{~s}^{-1}$ was applied. Data points taken at temperatures at which $j(T)<0.2 j\left(T_{\mathrm{m}}\right)$ were removed in this analysis. This procedure gave first approximation for the values of $\omega_{0 i}$ and $E_{i}(i=1,3,4)$. The program was then written to optimize the values of $\omega_{0 i}$ and $E_{i}(i=1,2,3,4)$ in order to provide a best fit between an experimental and predicted by Eqs. (6) to (9) TSD peaks. The values of $j(T)$ were calculated and the parameters were then varied systematically so as to minimize RSS, the weighted residual sum of squares, where

$$
\mathrm{RSS}=\sum_{i}\left\{\left[j_{i}(\text { calc })-j_{i}(\exp )\right] / j_{i}(\exp )\right\}^{2}
$$

$j_{i}$ means $j\left(T_{i}\right)$. The parameter values giving the best fit are

$$
\begin{array}{ll}
E_{1}=0.68 \mathrm{eV}, & \omega_{01}=(2.37 \pm 0.01) \times 10^{13} \quad\left[\mathrm{~s}^{-1}\right], \\
E_{2}=0.90 \mathrm{eV}, & \omega_{02}=(1.0 \pm 0.03) \times 10^{14} \quad\left[\mathrm{~s}^{-1}\right], \\
E_{3}=0.64 \mathrm{eV}, & \omega_{03}=(1.5 \pm 0.01) \times 10^{13} \quad\left[\mathrm{~s}^{-1}\right], \\
E_{4}=0.71 \mathrm{eV}, & \omega_{04}=(1.12 \pm 0.01) \times 10^{14} \quad\left[\mathrm{~s}^{-1}\right],
\end{array}
$$

The energy values are accurate to $\pm 0.01 \mathrm{eV}$. It should be noted that our values for the jump activation energies show a very good agreement with values $E_{1}=$ $0.69 \mathrm{eV}, E_{3}=0.65 \mathrm{eV}$, and $E_{4}=0.73 \mathrm{eV}$ reported in theoretical paper [26]. The value $E_{2}=0.90 \mathrm{eV}$ is in close agreement with $E_{2}=0.85 \mathrm{eV}$ given in [27]. The comparison of prediction and experimental data is presented in Fig. 1. As can be seen from this figure, the fitted curves are in close agreement with the experimental points.

Substitution of the parameter values listed above into Eqs. (8) and (9) gives $A_{1} / A_{2}=2.655 \times 10^{-2}$ for $T_{\mathrm{p}}=218 \mathrm{~K}$. The other feature is that $\left|\lambda_{2}(T)\right| \gg\left|\lambda_{1}(T)\right|$. Consequently, when temperature increases the current given by the second term in Eq. (6) rapidly decreases. For this reason the TSD bands are related mainly with the current given by the first term in Eq. (6). These facts prove the validity of approximation Eq. (11).

\section{Conclusion}

In summary, we can conclude that investigation of the reorientation of $\mathrm{I}-\mathrm{V}$ complexes with TSD method permits a number of parameters related to the move- 
ment of the vacancy in the nearest-neighbourhood and next-nearest neighbourhood of the impurity to be estimated.

\section{References}

[1] S. Radhakrishna, S. Haridos, Crystal Lattice Defects 7, 191 (1978).

[2] J. Vanderschueren, J. Gasiot, in: Topics in Applied Physics, Vol. 37, Ed. P. Braunlich, Springer-Verlag, Berlin 1979, Ch. 4.

[3] C. Bucci, R. Fieschi, Phys. Rev. 148, 816 (1966).

[4] Ah Mee Hor, P.W.M. Jacobs, Phys. Status Solidi A 44, 725 (1977).

[5] D.L. Kirk, R.M. Innes, J. Phys. C, Solid State Phys. 11, 1105 (1978).

[6] J.S. Dryden, R.G. Heydon, J. Phys. C, Solid State Phys. 11, 393 (1978).

[7] A. Wilbrand, F. Fischer, Phys. Status Solidi B 99, 243, 249 (1980).

[8] J. Prakash, A.K. Nishad, J. Rahul, Japan J. Appl. Phys. 25, 701 (1986).

[9] C. Vijayan, Y.V.G.S. Murti, Phys. Status Solidi A 105, 397 (1988).

[10] J. Kunze, P. Müller, Phys. Status Solidi 33, 91 (1969).

[11] A. Brun, P. Danas, F. Beniere, J. Phys. Chem. Solids 35, 249 (1974).

[12] A. Brun, P. Dansas, J. Phys. C, Solid State Phys. 7, 2593 (1974).

[13] F. Cussó, F. Jaque, Solid State Commun. 29, 283 (1979).

[14] A.B. Vassilikou, J. Grammatikakis, C.A. Londos, J. Phys. Chem. Solids 47, 727 (1986).

[15] V. Katsika, J. Grammatikakis, J. Phys. Chem. Solids 51, 1089 (1990).

[16] J. Poźniak, G. Berg, Phys. Status Solidi A 78, 69 (1983).

[17] J. Poźniak, J.K. Poźniak, Phys. Status Solidi B 200, 535 (1997).

[18] J. Poźniak, Phys. Status Solidi B 200, 265 (1997).

[19] H.F. Symmons, J. Phys. C, Solid State Phys. 3, 1846 (1970)); 4, 1945 (1971).

[20] J.S. Dryden, R.J. Meakins, Disc. Faraday Soc. 23, 39 (1957).

[21] P. Dansas, J. Phys. Chem. Solids 32, 2699 (1971).

[22] P. Varotsos, D. Miliotis, J. Phys. Chem. Solids 35, 927 (1974).

[23] R.W. Dreyfus, Phys. Rev. 121, 1675 (1961).

[24] J. Corish, P.B. Fitzsimons, P.W.M. Jacobs, Philos. Mag. A 56, 1 (1987).

[25] A.N. Murin, S.M. Banasevitch, Yu. Grushko, Sov. Phys.-Solid State 3, 1762 (1962).

[26] C.R.A. Catlow, J. Corish, J.M. Quigley, P.W.M. Jacobs, J. Phys. Chem. Solids 43, 231 (1980).

[27] H. Machida, W.J. Fredericks, J. Phys. (France) 37, C7-385 (1976). 